\title{
Erratum: Lattice relaxation and energy band modulation in twisted bilayer graphene [Phys. Rev. B 96, 075311 (2017)]
}

\author{
Nguyen N. T. Nam and Mikito Koshino
}

(Received 19 February 2020; accepted 20 February 2020; published 16 March 2020)

DOI: 10.1103/PhysRevB.101.099901

There is an error in the numerical calculation to solve the self-consistent equation [Eq. (33)] and in graphene's Lamé parameters. As a result, the strain vector $\mathbf{u}_{\mathbf{q}}^{-}$was underestimated in the original paper. In the following, we present the corrected tables and figures. These corrections, however, do not affect the qualitative argument and conclusions in the original paper. We adopt graphene's Lamé factors to $\lambda \approx 3.25$ and $\mu \approx 9.57 \mathrm{eV} / \AA^{2}[1,2]$. The $\eta$ parameters are updated as Table I. In numerically solving Eq. (33), we consider harmonics within radius $|\boldsymbol{q}| \leqslant 6 G_{\mathrm{M}}$. The corrected values of $\mathbf{u}_{\mathbf{q}}^{-}$are presented in Table II. Here, we set the $x y$ coordinates as in Fig. 1 such that $\mathbf{G}_{1}^{\mathrm{M}}=(1,0) G_{\mathrm{M}}$ and $\mathbf{G}_{2}^{\mathbf{M}}=(-1 / 2, \sqrt{3} / 2) G_{\mathrm{M}}$. Figures $8-10$ in the original paper are corrected as Figs. 2-4.

TABLE I. Index $(m, n)$, the rotation angle $\theta$, the size of the moiré unit-cell $L_{\mathrm{M}}$, and the dimensionless parameter $\eta$.

\begin{tabular}{llcr}
\hline \hline$(m, n)$ & $\theta\left(^{\circ}\right)$ & $L_{\mathrm{M}}(\mathrm{nm})$ & $\eta$ \\
\hline$(12,13)$ & 2.65 & 5.33 & 0.242 \\
$(22,23)$ & 1.47 & 9.59 & 0.436 \\
$(27,28)$ & 1.20 & 11.72 & 0.533 \\
$(31,32)$ & 1.05 & 13.42 & 0.610 \\
$(33,34)$ & 0.987 & 14.27 & 0.649 \\
$(40,41)$ & 0.817 & 17.26 & 0.784 \\
$(60,61)$ & 0.547 & 25.78 & 1.172 \\
\hline
\end{tabular}

TABLE II. Fourier components $i \mathbf{u}_{\mathbf{q}}^{-}=i\left(u_{\mathbf{q}}^{x-}, u_{\mathbf{q}}^{y-}\right)$ (in units of $a$ ) at wave-points $\mathbf{q}=m_{1} \mathbf{G}_{1}^{\mathrm{M}}+m_{2} \mathbf{G}_{2}^{\mathrm{M}}$ for the several rotation angles.

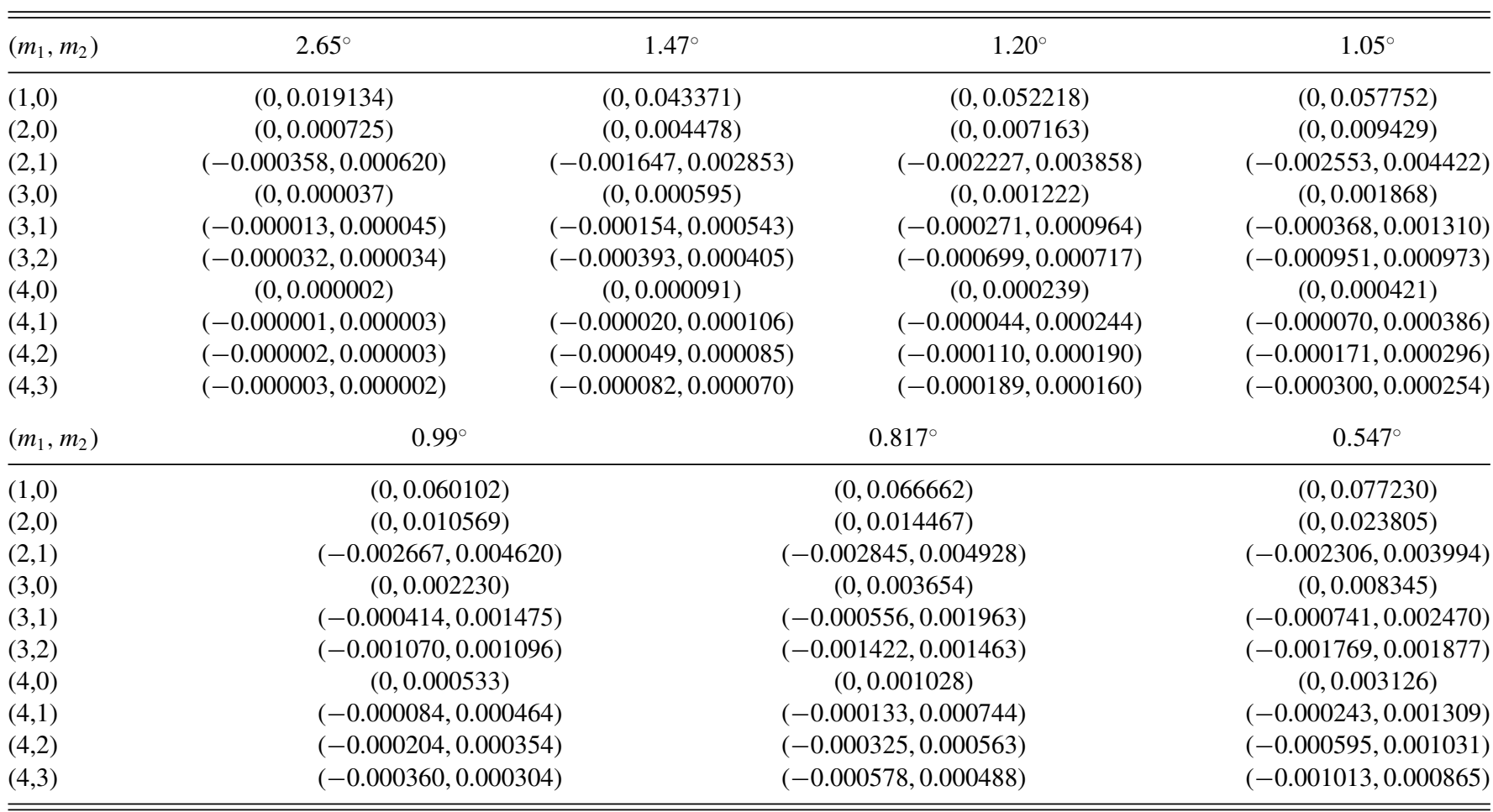




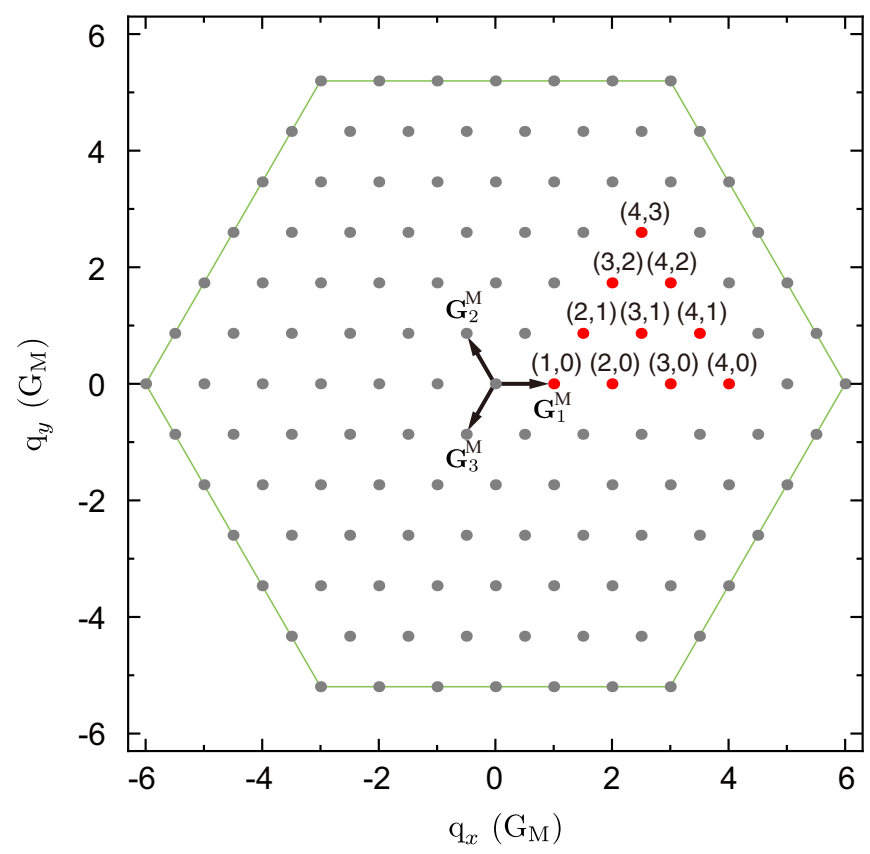

FIG. 1. (Updated version of Fig. 11.) The $\mathbf{q}$ points within radius $6 G_{M}$ in the reciprocal space. The red dots indicate the points chosen in Table II. 
(a) Displacement distance
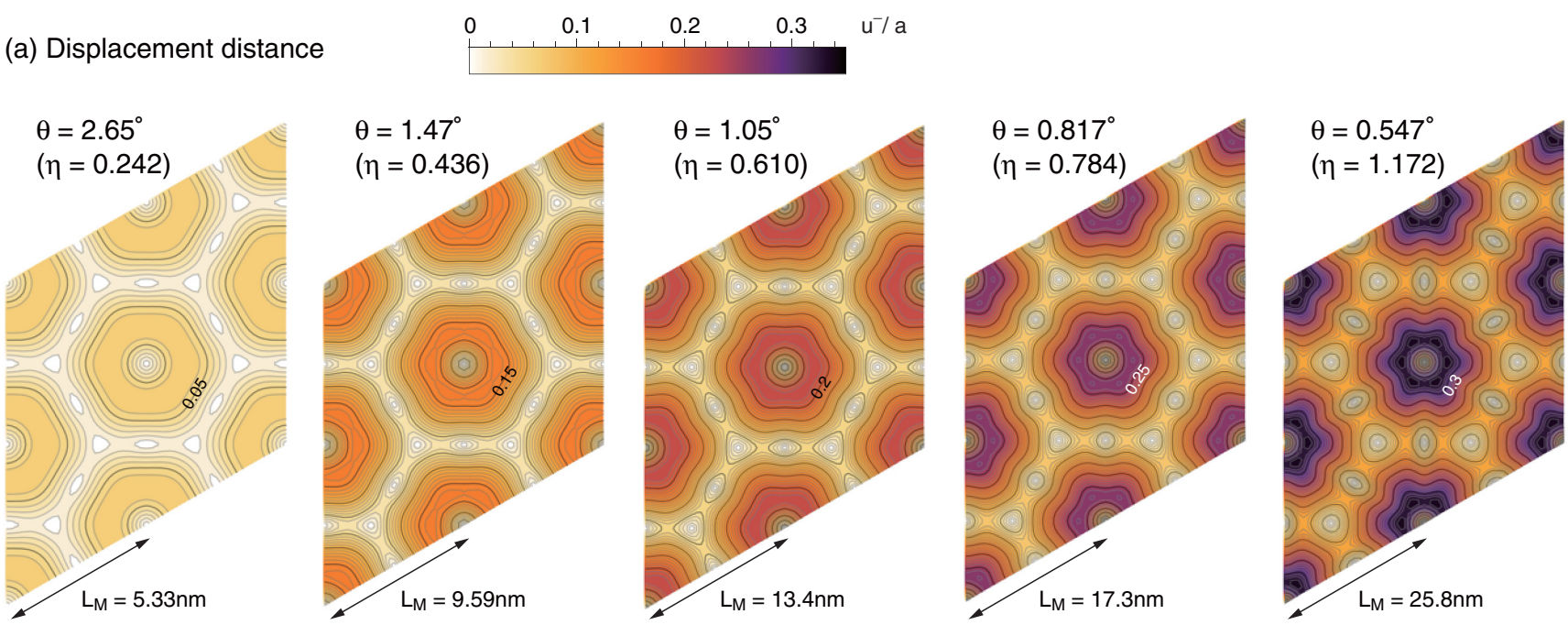

(b) Local binding energy
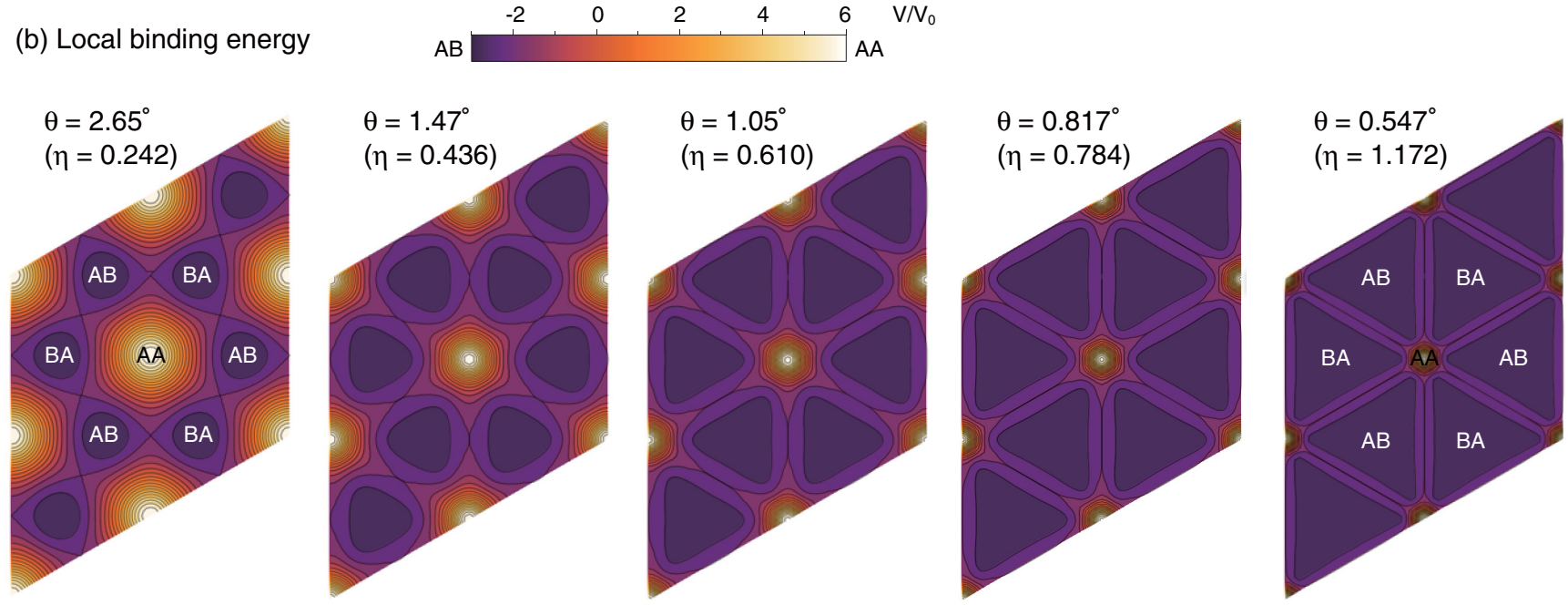

(c) Pseudo magnetic field

$\begin{array}{llllllll}-150 & -100 & -50 & 0 & 50 & 100 & 150 & \mathrm{~B}(\text { Tesla })\end{array}$
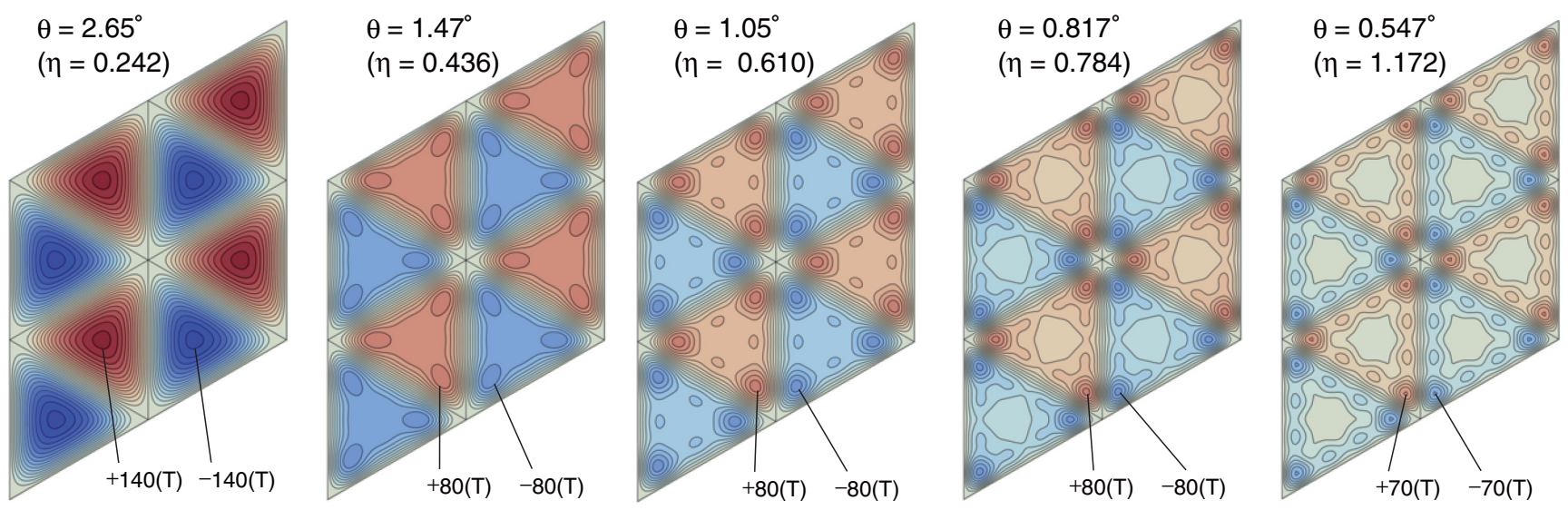

FIG. 2. (Updated version of Fig. 8.) Two-dimensional maps for (a) absolute value of the displacement vector $\mathbf{u}^{-}(\mathbf{r})$, (b) the local binding energy $V[\delta(\mathbf{r})]$, and (c) strain-induced pseudo-magnetic-field $B_{\text {eff }}(\mathbf{r})$, calculated for twisted bilayer graphenes (TBGs) with various rotation angles. 
(a) $\theta=2.65^{\circ}$

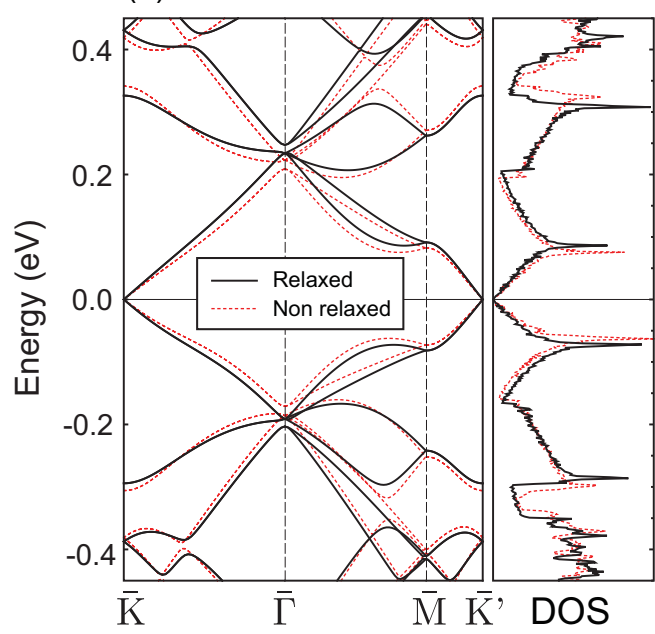

(c) $\theta=1.20^{\circ}$

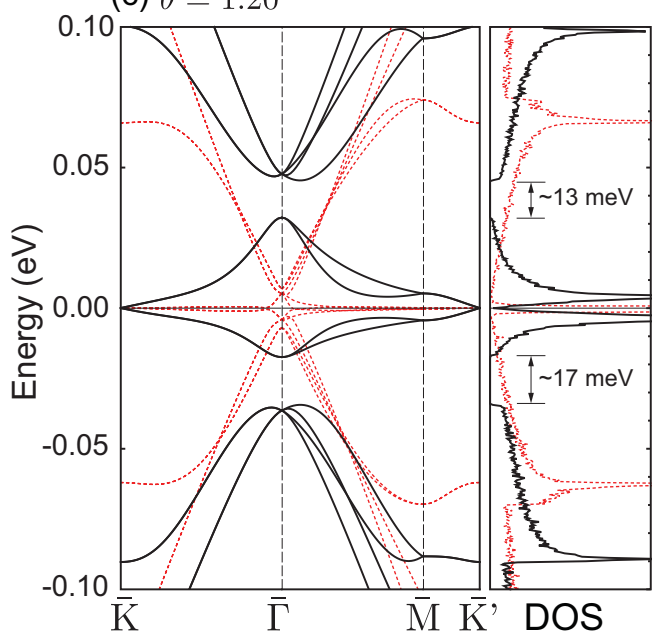

(e) $\theta=0.99^{\circ}$

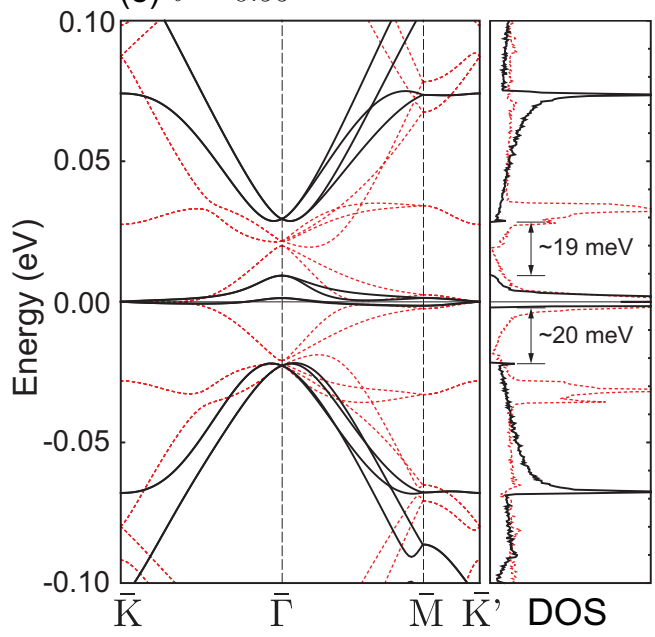

(b) $\theta=1.47^{\circ}$
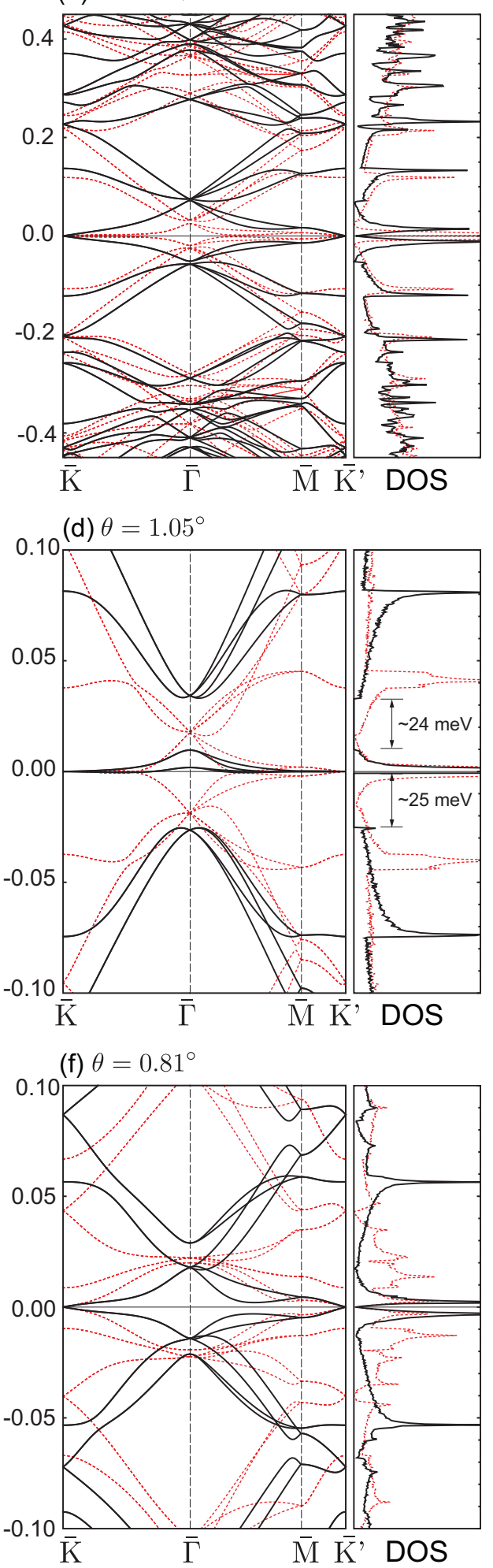

FIG. 3. (Updated version of Fig. 9.) Band structure and density of state (DOS) of relaxed (black solid lines) and nonrelaxed (red dashed lines) TBGs at various rotation angles. The energy gap is indicated by a pair of arrows in the DOS panel. 

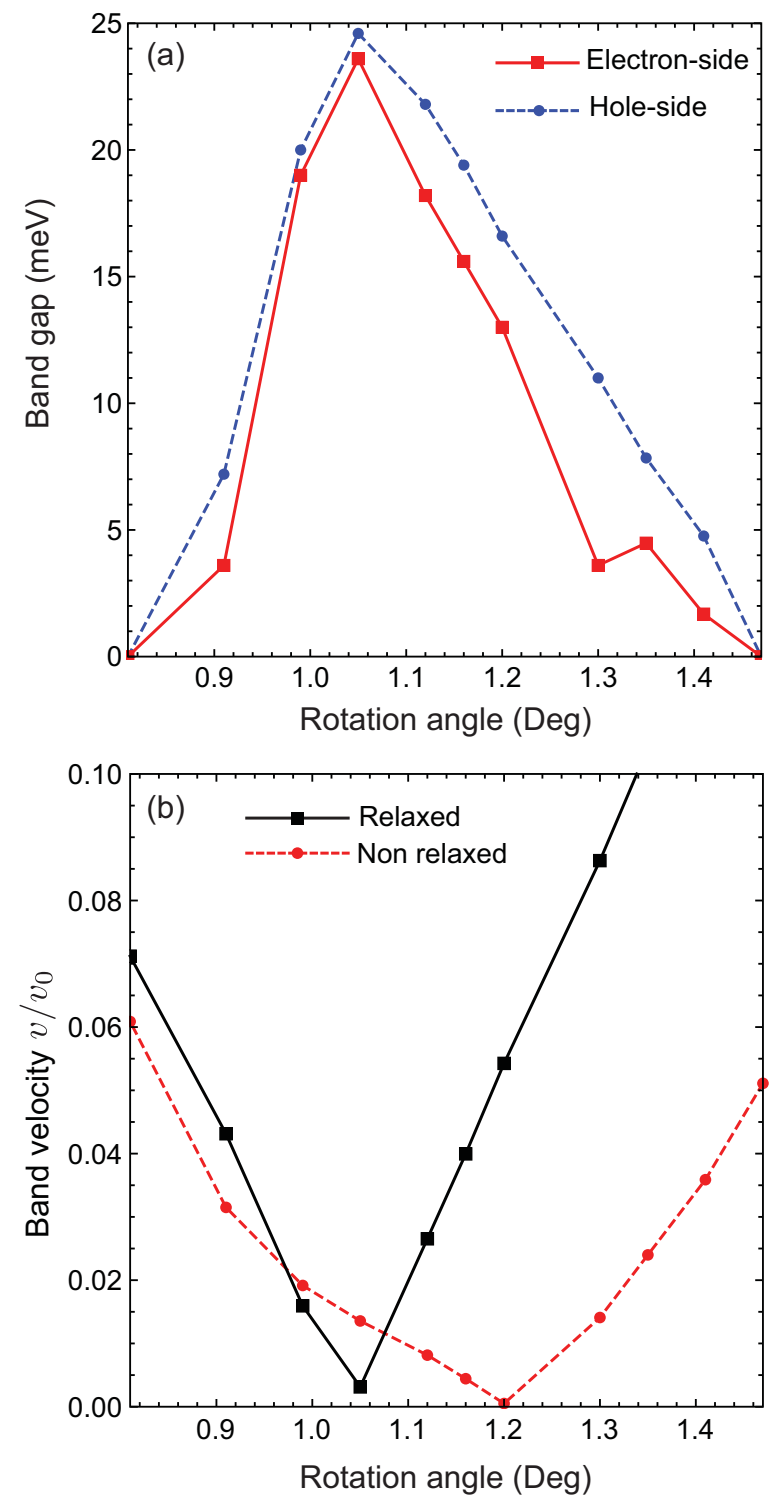

FIG. 4. (Updated version of Fig. 10.) (a) Band gap between the lowest band and the first excited bands on the electron's side (red, solid line) and on the hole's side (blue, dashed line) in relaxed TBGs against the rotation angle. (b) Band velocity at the $K$ point as a function of $\theta$ for relaxed (black, solid line) and nonrelaxed (red, dashed line) TBGs.

[1] K. Zakharchenko, M. Katsnelson, and A. Fasolino, Phys. Rev. Lett. 102, 046808 (2009).
[2] J. Jung, A. M. DaSilva, A. H. MacDonald, and S. Adam, Nat. Commun. 6, 6308 (2015). 